\title{
ORIGINAL ARTICLE \\ Bridging the gap between marker-assisted and genomic selection of heading time and plant height in hybrid wheat
}

\begin{abstract}
Y Zhao ${ }^{1,3}$, MF Mette ${ }^{1,3}$, M Gowda $^{2,4}$, CFH Longin ${ }^{2}$ and JC Reif ${ }^{1}$
Based on data from field trials with a large collection of 135 elite winter wheat inbred lines and $1604 \mathrm{~F}_{1}$ hybrids derived from them, we compared the accuracy of prediction of marker-assisted selection and current genomic selection approaches for the model traits heading time and plant height in a cross-validation approach. For heading time, the high accuracy seen with marker-assisted selection severely dropped with genomic selection approaches RR-BLUP (ridge regression best linear unbiased prediction) and BayesC $\pi$, whereas for plant height, accuracy was low with marker-assisted selection as well as RR-BLUP and BayesC $\pi$. Differences in the linkage disequilibrium structure of the functional and single-nucleotide polymorphism markers relevant for the two traits were identified in a simulation study as a likely explanation for the different trends in accuracies of prediction. A new genomic selection approach, weighted best linear unbiased prediction (W-BLUP), designed to treat the effects of known functional markers more appropriately, proved to increase the accuracy of prediction for both traits and thus closes the gap between marker-assisted and genomic selection.
\end{abstract}

Heredity (2014) 112, 638-645; doi:10.1038/hdy.2014.1; published online 12 February 2014

Keywords: marker-assisted selection; genomic selection; heading time; plant height; hybrid wheat

\section{INTRODUCTION}

Functional markers linked to quantitative trait loci (QTLs) are routinely used to predict the performance of important traits in domestic animals (Goddard and Hayes, 2009) and crop plants (Bernardo, 2008). Nevertheless, marker-assisted selection has its limitations (Heffner et al., 2009), as it is efficient only if the trait under consideration is controlled by a limited number of QTLs with large contributions to phenotypic variation, but is inferior to traditional phenotypic selection in dealing with complex agronomic traits controlled by many QTLs with small effects (Bernardo, 2001). One major reason is that estimates of QTL effects for minor QTLs are often biased.

As a solution for the prediction of performance in complex traits, genomic selection has been suggested (Meuwissen et al., 2001). In genomic selection, large numbers of markers are included and their effects are estimated in populations that have been genotyped and phenotyped. The estimated marker effects are then applied to predict the breeding value of nonphenotyped individuals based on their molecular marker profiles. The great potential of genomic selection for complex traits has been demonstrated in several experimental studies in plant and animal breeding populations (Bernardo, 2008; Heffner et al., 2009; Heslot et al., 2012; Massman et al., 2013).

One crucial challenge in genomic selection is to choose the appropriate biometrical model (Heffner et al., 2009; Heslot et al., 2012). The relative performance of biometrical models is expected to depend on the genetic architecture of the traits under scrutiny. In a recent simulation study, Clark et al. (2011) observed substantial higher accuracies of prediction for BayesB in comparison with RRBLUP (ridge regression best linear unbiased prediction) in a scenario assuming 100 QTLs underlying the trait under consideration. Whereas RR-BLUP is based on the infinitesimal model, BayesB implies that only a defined fraction of single-nucleotide polymorphisms (SNPs) contributes to the genotypic variation of the trait under consideration. The reported superiority of BayesB is challenged by a study based on experimental data in maize that described only marginal differences in accuracies between biometrical models and did not observe an association with genetic architecture (Riedelsheimer et al., 2013). One explanation for the deviation observed between prediction accuracies based on simulation versus experimental data could be the presence of linkage disequilibrium (LD) in experimental setups that would also enable infinitesimal model-based approaches to appropriately portray a genetic architecture with large effect QTLs. Besides the superiority of specific genomic selection models, comparison of the accuracy of marker-assisted versus genomic selection is of interest for those traits with QTLs exhibiting large effects on the genotypic variation. To the best of our knowledge, however, such a comparison based on experimental data sets for traits with known large effects is lacking.

Wheat is an important crop in which extensive studies of genetic architecture have been performed (Le Couviour et al., 2011; Würschum et al., 2013). Heading time and plant height are important traits for wheat production (Borlaug, 1983; Worland et al., 1998; Distelfeld et al., 2009) and key genes such as Ppd-D1, Rht-B1 and Rht-D1 controlling these traits have been characterized at the

${ }^{1}$ Department of Cytogenetics and Genome Analysis, Leibniz Institute of Plant Genetics and Crop Plant Research (IPK), Gatersleben, Germany and ${ }^{2}$ State Plant Breeding Institute, University of Hohenheim, Stuttgart, Germany

${ }^{3}$ These authors contributed equally to this work.

${ }^{4}$ Current address: International Maize and Wheat Improvement Center (CIMMYT), Nairobi, Kenya.

Correspondence: Professor JC Reif, Department of Cytogenetics and Genome Analysis, Leibniz Institute of Plant Genetics and Crop Plant Research (IPK), Corrensstraße 3 , 06466 Gatersleben, Germany.

E-mail: reif@ipk-gatersleben.de

Received 15 August 2013; revised 6 December 2013; accepted 13 December 2013; published online 12 February 2014 
molecular level. $P p d-D 1$ encoding a pseudo-response regulator family member (Turner et al., 2005; Beales et al., 2007) is a major regulator of photoperiod response in wheat with also some effect on plant height (Worland et al., 1998), whereas Rht-B1 and Rht-D1 encoding gibberellin response modulators are major regulators of plant height (Peng et al., 1999). The photoperiod-insensitive allele Ppd-D1a promoting early flowering and short growth as well as the gibberellininsensitive alleles $R h t-B 1 b$ or $R h t-D 1 b$ promoting semi-dwarf stature are well represented in wheat adapted to temperate zones (Guo et al., 2010; Le Couviour et al., 2011; Seki et al., 2011; Wilhelm et al., 2013a,b). Nevertheless, there is room for additional QTLs, making heading time and in particular plant height interesting model traits for a comparative study of the predictability of combined known major and unknown minor effects.

Based on phenotypic data obtained in four environments (Longin et al., 2013) and genotypic data generated using a wheat 9k SNP array (Akhunov et al., 2009; Cavanagh et al., 2013) and functional markers at gene loci Ppd-D1, Rht-B1 and Rht-D1 (Ellis et al., 2002; Beales et al., 2007) for a large collection of 135 elite winter wheat inbred lines and $1604 \mathrm{~F}_{1}$ hybrids derived from them, we compared the accuracy of prediction of marker-assisted selection and current genomic selection approaches for the model traits heading time and plant height in a cross-validation approach. For heading time, accuracy was high for marker-assisted selection and low for genomic selection using RRBLUP and BayesC $\pi$, whereas for plant height, accuracy was low for marker-assisted selection as well as for RR-BLUP and BayesC $\pi$. As a likely explanation for the different accuracies of prediction achieved for the two traits, differences in the LD structure of related functional and SNP markers were identified in a simulation study. We developed a weighted best linear unbiased prediction (W-BLUP) approach as a new genomic selection model with improved accuracy because of a more appropriate treatment of known functional markers. The W-BLUP approach efficiently bridges the gap between marker-assisted and genomic selection.

\section{MATERIALS AND METHODS}

\section{Plant materials and field trials}

Initially, we had sampled 68 potential male elite inbred lines with known good pollination characters and 275 potential female elite lines adapted to Central Europe and fingerprinted them with 24 simple sequence repeat (SSR) markers (Longin et al., 2013). Eliminating close relatives and maximizing the allelic diversity based on the simple sequence repeat marker profiles (while retaining $>77 \%$ of simple sequence repeat alleles present in the starting set of 343 lines), 15 male and 120 female parental lines were selected for hybrid production in a 15 times 120 factorial mating design using chemical hybridization agents (Longin et al., 2013). Sufficient $\mathrm{F}_{1}$ hybrid seeds were obtained for 1604 out of the 1800 potential single-cross hybrid combinations (Zhao et al., 2013b). All lines and hybrids were evaluated in 4 environments in 2012 together with 10 commercial checks (Longin et al., 2013). The environments were Böhnshausen (latitude $51^{\circ} 51^{\prime} \mathrm{N}$, longitude $10^{\circ} 57^{\prime} \mathrm{E}, 146 \mathrm{~m}$ above sea level (asl), sandy loam soil texture), Hadmersleben (latitude $51^{\circ} 59^{\prime} \mathrm{N}$, longitude $11^{\circ} 18^{\prime} \mathrm{E}, 88 \mathrm{~m}$ asl, silt loam soil texture), Harzhof (latitude $54^{\circ} 24^{\prime} \mathrm{N}$, longitude $9^{\circ} 51^{\prime} \mathrm{E}, 25 \mathrm{~m}$ asl, sandy loam soil texture) and Hohenheim (latitude $48^{\circ} 42^{\prime} \mathrm{N}$, longitude $9^{\circ} 12^{\prime} \mathrm{E}$, $390 \mathrm{~m}$ asl, sandy loam soil texture). The experimental designs were partially replicated $\alpha$-designs (Williams et al., 2011), where all parents, checks as well as $29 \%$ of the hybrids were used in both replications (for details, see Longin et al., 2013). Sowing density ranged from 230 to 290 seeds per $\mathrm{m}^{2}$ and plot size ranged from 7.5 to $9.7 \mathrm{~m}^{2}$. The trials were treated with fertilizers, fungicides and herbicides according to standard agronomic practices for intensive wheat production. Heading time was recorded as the number of days from 1 January to the day when half of the heads had emerged from flag leaves; plant height was measured in $\mathrm{cm}$ at one time point after heading in each environment.

\section{Phenotypic data analyses}

Phenotypic data were analyzed in two steps. First, we estimated the adjusted entry means for each location (for details, see Zhao et al., 2013b). In a second step, adjusted entry means were used to estimate the genetic variance components of hybrids and parental lines as well as the variance of genotype $\times$ location interactions. We followed the suggestion of Möhring and Piepho (2009) and weighted each observation with one divided by the squared standard error. Significance of variance component estimates were tested by model comparison with likelihood ratio tests where the halved $P$-values were used as an approximation (Stram and Lee, 1994). In addition, we assumed fixed genetic effects and estimated the best linear unbiased estimates of the 1739 genotypes. The phenotypic data analyses were performed using the software ASReml-R 3.0 (Butler et al., 2009).

\section{Genotypic data analysis}

Genotyping was done with a 9k SNP array based on the Illumina Infinium assay (Cavanagh et al., 2013) (Illumina, San Diego, CA, USA). After excluding SNP markers with (1) a rate of missing values above $5 \%$, (2) a rate of heterozygosity above $5 \%$ or (3) a minor allele frequency of $<0.05$ (Miedaner et al., 2012), in total 1280 SNP markers were retained (Supplementary Table S1). In addition, in genes Ppd-D1 (Beales et al., 2007), Rht-B1 and Rht-D1 (Ellis et al., 2002), one SNP each was analyzed as a functional marker using LGC Genomics KASP assays (LGC Genomics, Berlin, Germany). Missing genotypes were imputed following the approach suggested by Crossa et al. (2010).

\section{Marker-assisted selection}

The functional marker for Ppd-D1 (Beales et al., 2007) is used as a standard tool in European wheat breeding, explaining up to $30 \%$ of the genotypic variation of heading time. In addition, the functional markers for Ppd-D1 (Beales et al., 2007) as well as Rht-B1 and Rht-D1 (Ellis et al., 2002) are used routinely in wheat breeding in Europe, explaining up to $40 \%$ of the genotypic variation for plant height. We defined the design matrices for the additive and dominance effects of the three functional markers according to the $\mathrm{F}_{\infty}$ metric of Falconer and Mackay (1996) and used a standard multiple regression approach to estimate their effects in the germplasm under consideration. A general term was fitted to groups of females, males and hybrids for plant height to take into account heterosis for this trait, whereas no such correction was needed for heading time in the absence of heterosis (Longin et al., 2013). Furthermore, we used a step-wise backward selection based on the Akaike information criterion to test for relevant two-way epistatic interactions using the step function (Venables and Ripley, 2002) implemented in the software package R (R Core Team, 2012).

\section{Genomic selection}

Based on the adjusted entry means of the 1739 genotypes, we applied three approaches for genomic selection considering additive and dominance effects: RR-BLUP (Whittaker et al., 2000), BayesC $\pi$ (Dekkers et al., 2009; Habier et al., 2011) and a newly developed modification of RR-BLUP using specific weights for the known functional markers denoted as W-BLUP. All statistical procedures for the genomic selection approaches were executed using R (R Core Team, 2012). Details of the implementation of the RR-BLUP and BayesC $\pi$ models have been described in Zhao et al. (2013a). Briefly, the general form of the three models is defined as the following:

$$
Y=1_{n} \mu+Z_{A} a+Z_{D} d+e,
$$

where $Y$ are the adjusted entry means of the 1739 genotypes across the four locations, $l_{n}$ is a vector of ones and $n$ is the number of genotypes; $\mu$ refers to the overall mean across all four locations; $a$ is the additive marker effect and $d$ is the dominance marker effect. $Z_{A}$ and $Z_{D}$ are design matrices for the additive and dominance effects of the markers specified according to the $\mathrm{F}_{\infty}$ metric of Falconer and Mackay (1996) and $e$ is the residual. 
RR-BLUP. For the RR-BLUP model, we assume that additive and dominance marker effects have normal distributions $N\left(0, \sigma_{a}^{2}\right)$ and $N\left(0, \sigma_{d}^{2}\right)$ with constant variances of additive effects $\sigma_{a}^{2}$ and dominance effects $\sigma_{d}^{2}$. The estimates of $\mu$, $a$ and $d$, which denote as $\hat{\mu}, \hat{a}$ and $\hat{d}$, were obtained from the following mixed-model equation (Henderson, 1984):

$$
\left[\begin{array}{c}
\hat{\mu} \\
\hat{a} \\
\hat{d}
\end{array}\right]=\left[\begin{array}{ccc}
1_{n}^{T} 1_{n} & 1_{n}^{T} Z_{A} & 1_{n}^{T} Z_{D} \\
Z_{A}^{T} 1_{n} & Z_{A}^{T} Z_{A}+\lambda_{A} I_{m} & Z_{A}^{T} Z_{D} \\
Z_{D}^{T} 1_{n} & Z_{D}^{T} Z_{A} & Z_{A}^{T} Z_{D}+\lambda_{D} I_{m}
\end{array}\right]^{-1}\left[\begin{array}{c}
1_{n}^{T} Y \\
Z_{A}^{T} Y \\
Z_{D}^{T} Y
\end{array}\right]
$$

Here, $I_{m}$ refers to an identity matrix with dimension of $m$, where $m$ is the number of markers. The shrinkage parameters $\lambda_{A}$ and $\lambda_{D}$ are defined as the ratios between the variance of residuals and the variance of the marker effects (Meuwissen et al., 2001). Required variance components were estimated based on adjusted entry means of individual environments decomposing the variance of hybrids into variance due to general and variance due to specific combining ability effects (Hallauer and Miranda, 1988).

BayesC $\pi$. Whereas in RR-BLUP it is assumed that all markers contribute to genetic variance, in BayesC $\pi$ only a fraction $1-\pi_{g}(g$ denotes either $a$ or $d)$ of the used markers is considered to contribute to the genetic variance. Based on this assumption, the model for BayesC $\pi$ is:

$$
Y=1_{n} \mu+Z_{A} \delta_{a} a+Z_{D} \delta_{d} d+e
$$

The additional parameter $\delta_{g}$ has a prior distribution:

$$
\delta_{g} \sim\left\{\begin{array}{c}
0, \text { with probability } \pi_{g} \\
1, \text { with probability } 1-\pi_{g}
\end{array}\right.
$$

In BayesC $\pi$, a uniform $(0,1)$ prior was assumed for $\pi_{g}$, resulting in a $\beta$-distribution for the full-conditional posterior (Habier et al., 2011). For BayesC $\pi$, all above outlined parameters have to be sampled from their fullconditional posterior using a special Markov chain Monte Carlo method called Gibbs sampling.

The overall mean $\mu$ is sampled from a normal distribution $\mu \sim N\left(\frac{1_{n}^{T}\left(y-Z_{A} \delta_{a} a-Z_{D} \delta_{d} d\right)}{n}, \frac{\sigma_{e}^{2}}{n}\right)$ The variance of residual and additive effects are sampled from the inverted $\chi^{2}$ distribution:

$\sigma_{\mathrm{e}}^{2} \sim\left(\mathrm{e}^{\mathrm{T}} \mathrm{e}+v_{\mathrm{e}} \mathrm{S}_{\mathrm{e}}^{2}\right) \chi_{v_{\mathrm{e}}+\mathrm{n}}^{-2}$, and $\sigma_{\mathrm{a}}^{2} \sim\left(\mathrm{a}^{\mathrm{T}} \mathrm{a}+v_{\mathrm{a}} \mathrm{S}_{\mathrm{a}}^{2}\right) \chi_{v_{\mathrm{e}}+\mathrm{m}(\mathrm{t})}^{-2}$. Here, $m(t)$ refers to the number of non-zero additive effects in $t$-th Markov chain Monte Carlo iteration. The variance of dominance effects is sampled from the inverted $\chi^{2}$ square distribution: $\left[\left(\mathrm{d}-\mu_{d}\right)^{\mathrm{T}}\left(\mathrm{d}-\mu_{d}\right)+v_{\mathrm{d}} \mathrm{S}_{\mathrm{d}}^{2}\right] \chi_{v_{\mathrm{d}}+\mathrm{m}(\mathrm{t})}^{-2}$ where $\mu_{d}$ is the expected mean for $d_{i}$, and $m(t)$ refers to the number of non-zero dominance effects in $t$-th Markov chain Monte Carlo iteration. In the above, the fixed parameters $v_{e}=v_{a}=v_{d}=4$, whereas $S_{e}^{2}$, $S_{a}^{2}$ and $S_{d}^{2}$ were calculated by the genetic variance approach we used in the above RR-BLUP model.

For $i$-th marker effects $a_{i}$ and $d_{i}$, we sampled new $a_{i}$ and $d_{i}$ from a full-conditional posterior $\left(a_{i}\right)_{\text {new }} \sim N\left(\frac{Z_{A_{i}}^{T}\left(Z_{A_{i}} a_{i}+e\right)}{Z_{A_{i}}^{T} Z_{A_{i}}+\frac{\sigma_{e}^{2}}{c}}, \frac{\sigma_{e}^{2}}{Z_{A_{i}^{T}}^{T} Z_{A_{i}}+\frac{\sigma_{e}^{2}}{c}}\right), \quad$ and $\left(d_{i}\right)_{\text {new }} \sim \mathrm{N}\left(\frac{\mathrm{Z}_{\mathrm{D}_{\mathrm{i}}}^{\mathrm{T}}\left(\mathrm{Z}_{\mathrm{D}_{\mathrm{i}}} \mathrm{d}_{\mathrm{i}}+\mathrm{e}\right)+\mu_{\mathrm{d}} \frac{\sigma_{e}^{2}}{\sigma_{\mathrm{d}}^{2}}}{Z_{\mathrm{D}_{\mathrm{i}}}^{\mathrm{T}} Z_{\mathrm{D}_{\mathrm{i}}}+\frac{\sigma_{e}^{2}}{\sigma_{\mathrm{d}}^{2}}}, \frac{\sigma_{e}^{2}}{Z_{\mathrm{D}_{\mathrm{i}}}^{\mathrm{T}} Z_{\mathrm{D}_{\mathrm{i}}}+\frac{\sigma_{e}^{2}}{\sigma_{\mathrm{d}}^{2}}}\right)$, where $Z_{\mathrm{A}_{\mathrm{i}}}, Z_{\mathrm{D}_{\mathrm{i}}}$ refers to the $i$-th column of $Z_{A}$ and $Z_{D}$. Note that a new $a_{i}$ and $d_{i}$ was only accepted with probability $\frac{1-\pi_{\mathrm{a}}}{1-\pi_{\mathrm{a}}+\mathrm{p}_{\mathrm{a}} \pi_{\mathrm{a}}}$ and $\frac{1-\pi_{\mathrm{d}}}{1-\pi_{\mathrm{d}}+\mathrm{p}_{\mathrm{d}_{\mathrm{d}}} \pi_{\mathrm{d}}}$, where $\mathrm{p}_{\mathrm{a}_{\mathrm{i}}}$ was the ratio of likelihood with $\delta_{\mathrm{a}_{\mathrm{i}}}=0$ and $\delta_{\mathrm{a}_{\mathrm{i}}}=1$, and $\mathrm{p}_{\mathrm{d}_{\mathrm{i}}}$ was the ratio of likelihood with $\delta_{\mathrm{d}_{\mathrm{i}}}=0$ and $\delta_{\mathrm{d}_{\mathrm{i}}}=1$.

After renewing all the parameters above, the $\pi_{g}(g=a$ or $d)$ used for the next iteration was updated with a $\beta$-distribution $\pi_{\mathrm{g}} \sim \operatorname{Beta}\left(1, \mathrm{~m}-\delta_{\mathrm{g}}^{\mathrm{T}} \delta_{\mathrm{g}}+1, \delta_{\mathrm{g}}^{\mathrm{T}} \delta_{\mathrm{g}}+1\right)$.

The above Gibbs sampling was run for 10000 times, and the first 1000 cycles were discarded as burn in

W-BLUP. The model used in W-BLUP is similar to the BLUP model, but we added an additional effect for the functional markers:

$Y=1_{n} \mu+Z_{A} a+F_{A} a_{f}+Z_{D} d+F_{D} d_{f}+e$, where $a_{f}, d_{f}$ denote the additive and dominance effects of the functional markers, and $F_{A}$ and $F_{D}$ are the design matrices for them. Thus, the mixed model equation for this model will be:

$$
\left[\begin{array}{c}
\hat{\mu} \\
\widehat{a} \\
\widehat{a_{f}} \\
\hat{d} \\
\widehat{d_{f}}
\end{array}\right]=\left[\begin{array}{ccccc}
1_{n}^{T} 1_{n} & 1_{n}^{T} Z_{A} & 1_{n}^{T} F_{A} & 1_{n}^{T} Z_{D} & 1_{n}^{T} F_{D} \\
Z_{A}^{T} 1_{n} & Z_{A}^{T} Z_{A}+\lambda_{A} I_{m} & Z_{A}^{T} F_{A} & Z_{A}^{T} Z_{D} & Z_{A}^{T} F_{D} \\
F_{A}^{T} 1_{n} & F_{A}^{T} Z_{A} & F_{A}^{T} F_{A}+\lambda_{A f} I_{m_{f}} & F_{A}^{T} Z_{D} & F_{A}^{T} F_{D} \\
Z_{D}^{T} 1_{n} & Z_{D}^{T} Z_{A} & Z_{D}^{T} F_{A} & Z_{D}^{T} Z_{D}+\lambda_{D} I_{m} & Z_{D}^{T} F_{D} \\
F_{D}^{T} 1_{n} & F_{D}^{T} Z_{A} & F_{D}^{T} F_{A} & F_{D}^{T} Z_{D} & F_{D}^{T} F_{D}+\lambda_{D_{f}} I_{m f}
\end{array}\right]^{-1}\left[\begin{array}{c}
1_{n}^{T} y \\
Z_{A}^{T} y \\
F_{A}^{T} y \\
Z_{D}^{T} y \\
F_{D}^{T} y
\end{array}\right]
$$

Here, $I_{m_{f}}$ refers to an identity matrix, whereas $m_{f}$ is the number of functional markers. The shrinkage parameters $\lambda_{A_{f}}$ and $\lambda_{D_{f}}$ are now defined as the ratio between the variance of residuals and the variance of the functional marker effects estimated by using marker-assisted selection in each training set. In this way, we give in particular a larger weight to the functional markers than to the general markers.

Hybrid performance was predicted based on the estimated additive and dominance effects (Zhao et al., 2013a). To study the influence of dominance effects on the prediction accuracy, we estimated the hybrid performance based solely on the additive or dominance effects.

\section{Cross-validations}

We evaluated the accuracy of prediction of heading time and plant height by genomic selection with the two established approaches RR-BLUP and BayesC $\pi$ as well as the newly developed W-BLUP using cross-validation. As population structure in factorial crosses strongly influences prediction accuracy, we used a cross-validation strategy in which training and validation were performed in sets that were not related via shared parental lines. We sampled 100 times 10 male and 80 female parental lines plus 610 hybrids derived from them as training set and estimated the additive and dominance effects. Only hybrids originating from the remaining 5 male and 40 female parental lines formed the validation set in which predictions derived from the training set were tested for their cross-validation accuracy. Prediction accuracy was estimated as Pearson's correlation coefficient between the observed and the predicted hybrid performance.

\section{Evaluation of accuracy with computer simulations}

We investigated the accuracy to predict the phenotypic performance by conducting computer simulations based on the marker data of our study. We followed the suggestion of Perez et al. (2010) and performed calculations based on the assumption that most markers have very small effects except for one marker that exhibits large additive and one marker that exhibits large dominance effects. We further assumed an average ratio of genetic variance explained by additive and dominance effects of 0.42 and a heritability of 0.77 . Moreover, the influence of the LD pattern on the prediction accuracy was approached by comparing simulations. Data set $L E-Q T L$, in which the selected marker of interest had no LD $\left(r^{2}\right.$ values $\left.<0.1\right)$ with all other markers involved, and data set $L D-Q T L$, in which the selected marker was set to show strong linkage disequilibrium with $r^{2}$ values $>0.7$ with at least 10 further markers, were designed based on representative SNPs from our study and employed in marker-assisted and genomic selection.

\section{RESULTS}

Phenotyping revealed broad genotypic variation for heading time and plant height

A set of 135 elite winter wheat inbred lines and $1604 \mathrm{~F}_{1}$ hybrids derived from them, adding up to in total 1739 entries, was evaluated for heading time and plant height in the field under natural settings at four locations. This revealed substantial genetic variation within the experimental population, and comparably high estimates of heritability of above 0.82 for heading time and plant height (Table 1).

As a prerequisite for efficient hybrid seed production, the 135 inbred lines had to be split into a group of 15 later-heading, taller, open-pollinating types to be used as male and a group of 120 earlier, shorter types to be used as female parents. This grouping is well apparent in the distribution of genotypic values among the male and female inbred lines (Figure 1). Mean average heading time and plant height, respectively, were 151 days and $86.6 \mathrm{~cm}$ for male and 149 days 
Table 1 Estimates of variance components $\left(\sigma^{2}\right)$ and heritability $\left(H^{2}\right)$ on entry mean basis for heading time (in days after 1 January) and plant height (in $\mathrm{cm}$ ) in 135 parental winter wheat inbred lines and their 1604 factorial crosses

\begin{tabular}{lcr}
\hline Source & Heading time & Plant height \\
\hline Parents & & \\
$\sigma^{2}$ Genotype & $3.58^{\mathrm{a}}$ & $22.54^{\mathrm{b}}$ \\
$\sigma^{2}$ Genotype $\times$ environment & $1.26^{\mathrm{a}}$ & $6.58^{\mathrm{b}}$ \\
$H^{2}$ & 0.87 & 0.85 \\
& & \\
$F_{1}$ hybrids $^{2}$ Genotype & & \\
$\sigma^{2}$ Genotype $\times$ environment & $2.04^{\mathrm{a}}$ & $17.79^{\mathrm{b}}$ \\
$\sigma^{2}$ Error & $0.58^{\mathrm{a}}$ & $5.39^{\mathrm{b}}$ \\
$H^{2}$ & 1.13 & 12.71 \\
& 0.85 & 0.82 \\
\hline
\end{tabular}

${ }^{a}$ Significantly different from zero at the 0.001 level of probability. bSignificantly different from zero at the 0.01 level of probability.

${ }^{c}$ Assumed same error variance for parents and $F_{1}$ hybrids.
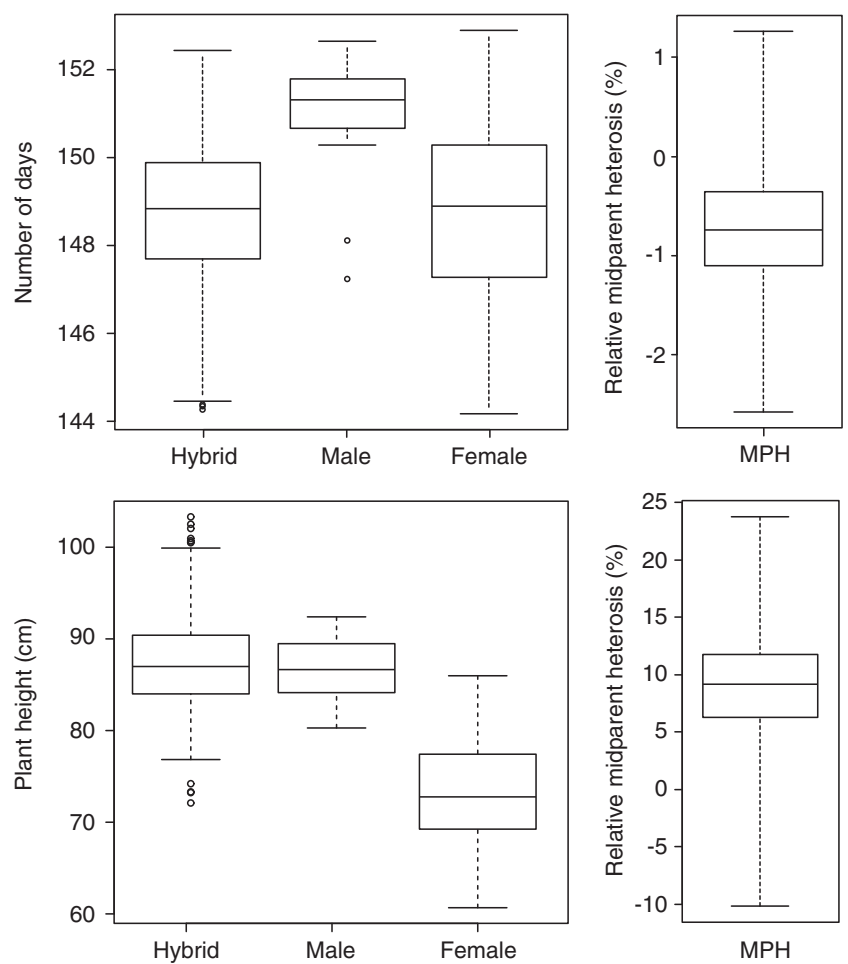

Figure 1 Box-and-whisker plots of the distribution of genotypic values for heading time and plant height for the 15 male and 120 female lines, and the resulting 1604 single-cross hybrids evaluated across four environments.

and $73.6 \mathrm{~cm}$ for female parental lines, in comparison with 149 days and $75.0 \mathrm{~cm}$ for all inbred lines together.

Alleles of major gene loci controlling heading time and plant height were distributed unevenly among male and female parental lines

The allele status of major gene loci controlling heading time and plant height was determined in parental inbred lines and hybrids. Locus $P p d-D 1$ is a major determinant of heading time in wheat, with the photoperiod-insensitive allele $P p d-D 1 a$ promoting early heading in temperate zones (Beales et al., 2007). All 15 late-heading inbred lines
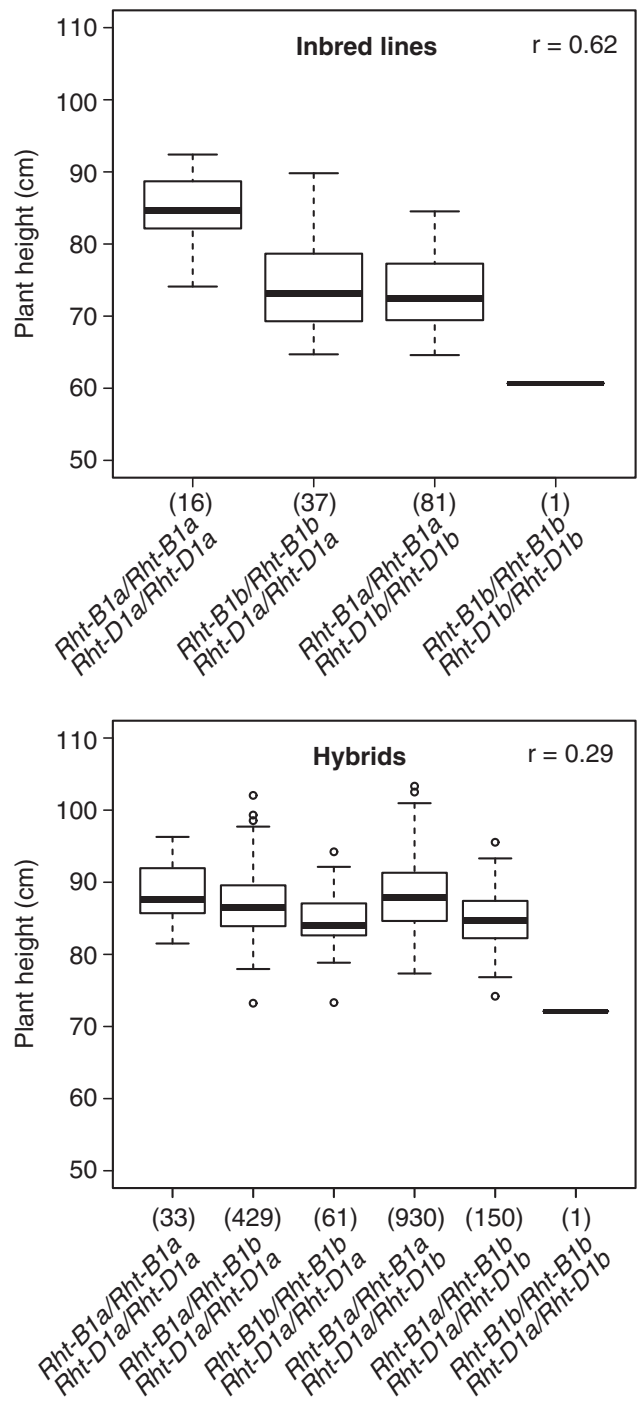

Figure 2 Box-and-whisker plots of the distribution of the genotypic values for plant height for different Rht-B1 and Rht-D1 allele setups among the 135 parent lines and 1604 single-cross hybrids. Whereas alleles $R h t-B 1 a$ and Rht-D1a promote tall plant stature, alleles Rht-B1b and RhtD1b promote dwarfing. Numbers in parentheses indicate numbers of inbred lines or hybrids with respective genotypes.

used as male parents were homozygous for the photoperiod-sensitive allele $P p d-D 1 b$, whereas among the earlier female parents, lines homozygous for either Ppd-D1a or Ppd-D1b were present.

Plant height is mainly influenced by loci $R h t-B 1$ and $R h t-D 1$, with alleles Rht-B1a and Rht-D1a promoting tall plant stature and alleles $R h t-B 1 b$ and Rht-D1b promoting dwarfing (Ellis et al., 2002). This is also reflected in our data (Figure 2). Inbred lines with allelic setup Rht-B1a/Rht-B1a;Rht-D1a/Rht-D1a were tallest with a median of average plant height of $84.6 \mathrm{~cm}$, followed by $R h t-B 1 b / R h t-B 1 b$; Rht-D1a/Rht-D1a and Rht-B1a/Rht-B1a;Rht-D1b/Rht-D1b with 73.2

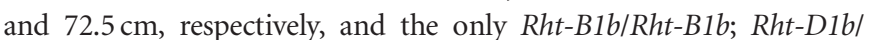
$R h t-D 1 b$ line included, with an average plant height of $60.7 \mathrm{~cm}$ (Figure 2). Among the tall lines used as male parents in hybrid production, 13 were of type Rht-B1a/Rht-B1a;Rht-D1a/Rht-D1a and two were of type Rht-B1b/Rht-B1b;Rht-D1a/Rht-D1a, whereas among inbred lines used as female parents, all four possible homozygous Rht-B1;Rht-D1 allele combinations were present. 
Hybrids displayed heterosis for plant height but not for heading time

We observed contrasting responses of the two traits of interest to hybridization (Figure 1). The median of average heading time of hybrids was more similar to that of the 120 earlier-heading inbred lines used as female parents, and average relative midparent heterosis of heading time was only $0.7 \%$. This low average midparent heterosis observed in our study can be explained by small dominance effects and/or by a lack of genetic differentiation among the male and female lines. In contrast, average relative midparent heterosis was substantial for plant height, amounting to $9.2 \%$. Thus, the median of average plant height of hybrids was more similar to that of the tall 15 inbred lines selected as male parents.

To further scrutinize the heterosis for plant height, hybrids were also grouped according to their allelic setup at gene loci Rht-B1 and $R h t-D 1$ (Figure 2). As only two of the possible four homozygous Rht$B 1 ; R h t-D 1$ allele combinations had been employed as male parents, only six of the possible nine Rht-B1;Rht-D1 allele combinations were present among the hybrids, including only two allowing direct comparison with parental inbred lines. For these two combinations, Rht-B1a/Rht-B1a;Rht-D1a/Rht-D1a and Rht-B1b/Rht-B1b;Rht-D1al Rht-Dla, hybrids were taller than the respective inbred lines, with medians of average plant height of $87.6 \mathrm{~cm}$ ( $+4 \%$ compared with inbred lines) and $84.0 \mathrm{~cm}(+15 \%)$, respectively (Figure 2$)$. Thus, heterosis of plant height manifested between inbred lines and hybrids carrying the same, homozygous Rht-B1;Rht-D1 allele setup.

As $P p d-D 1$ is also known to influence plant height (Worland et al., 1998; Beales et al., 2007), its allele status was checked in the compared plant groups. All Rht-B1a/Rht-B1a;Rht-D1a/Rht-D1a parental lines and hybrids that were included in our study were at the same time homozygous for the photoperiod-sensitive allele $P p d-D 1 b$ promoting a tall plant stature that excluded differences at this locus as an explanation for differences in plant height. Consistently, plant height heterosis between $R h t-B 1 b / R h t-B 1 b ; R h t-D 1 a / R h t-D 1 a$ inbred lines and hybrids persisted when subgroups containing only material homozygous for $P p d-D 1 b$ were compared.

\section{Marker-assisted selection for heading time and plant height}

The genotypic variation of heading time could be predicted by marker-assisted selection based on the functional marker for $P p d-D 1$ (Beales et al., 2007) with an accuracy of 0.45 for the parental inbred

Table 2 Average prediction accuracy of MAS and three different approaches of genomic selection for heading time and plant height

\begin{tabular}{|c|c|c|c|c|}
\hline \multirow[t]{2}{*}{ Method } & \multicolumn{2}{|c|}{ Heading time } & \multicolumn{2}{|c|}{ Plant height } \\
\hline & Accuracy & s.d. & Accuracy & s.d. \\
\hline MAS-NCVa & 0.526 & - & 0.444 & - \\
\hline MAS $^{b}$ & 0.535 & 0.091 & 0.378 & 0.101 \\
\hline RR-BLUPC & 0.404 & 0.112 & 0.395 & 0.135 \\
\hline BayesC $\pi^{\mathrm{c}}$ & 0.442 & 0.114 & 0.422 & 0.125 \\
\hline W-BLUPC & 0.576 & 0.110 & 0.502 & 0.115 \\
\hline
\end{tabular}

Abbreviations: MAS, marker-assisted selection; RR-BLUP, ridge regression best linear unbiased prediction; NCV, non-cross-validation; W-BLUP weighted best linear unbiased prediction; s.d., standard deviation.

MAS-NCV indicates MAS prediction accuracy without cross-validation based on functional marker $P p d-D 1$ for heading time and functional markers $P p d-D 1, R h t-B 1$ and $R h t-D 1$ for plant height. As only one value is available, s.d. could not be determined.

bMAS prediction accuracy according to cross-validation.

${ }^{c}$ Genomic selection accuracies according to cross-validation for RR-BLUP, BayesC $\pi$ and

W-BLUP based on 1280 markers plus the functional markers. lines (data not shown) and with an accuracy of 0.53 for the hybrids (Table 2). In the pooled populations of parental inbred lines and hybrids, prediction accuracy of marker-assisted selection for heading time amounted to a rather high 0.54 based only on the functional marker for $P p d-D 1$. The degree of dominance was -0.72 in the direction of the early-flowering homozygous Ppd-D1a class.

Prediction accuracy of marker-assisted selection for plant height based on the functional markers for Rht-B1 and Rht-D1 (Ellis et al., 2002) was much higher with 0.62 for the inbred lines as compared with 0.29 for the hybrids (Figure 2). Adding the functional marker information for Ppd-D1 (Beales et al., 2007) to the one for Rht-B1 and $R h t-D 1$ led to an increased prediction accuracy of 0.44 in the population of the hybrids. In the pooled populations of inbred lines and hybrids, the prediction accuracy amounted to 0.69 based on all three functional markers for gene loci $P p d-D 1, R h t-B 1$ and $R h t-D 1$. The degrees of dominance of the three functional marker loci for plant height determined on the basis of combined inbred line and hybrid data with fitting general terms to groups of female parents, male parents and hybrids were 1.2 toward the tall allele Rht-B1a, and 0.5 and 1.2 toward the short alleles Rht-D1b and Ppd-Dla, respectively (Figure 3 ). The model selection revealed significant digenic epistatic interactions involving additive and dominance effects among gene loci Rht-B1 and Rht-D1.

Cross-validated accuracy of marker-assisted and genomic selection The cross-validation of marker-assisted selection revealed no severe drop in prediction accuracies (Table 2) compared with the non-crossvalidated accuracies (Figure 2) for both heading time and plant height. This is not surprising as we considered the accuracy of marker-assisted selection based on well-established functional markers for plant height and heading time. The marginally higher accuracy for cross-validated versus non-cross-validated values observed for heading time resulted from the fact that the accuracies of prediction were evaluated exclusively in the population of hybrids. For the non-crossvalidated values, this applies to the estimation and the test set, as both are identical. In contrast, in the cross-validation study, we used

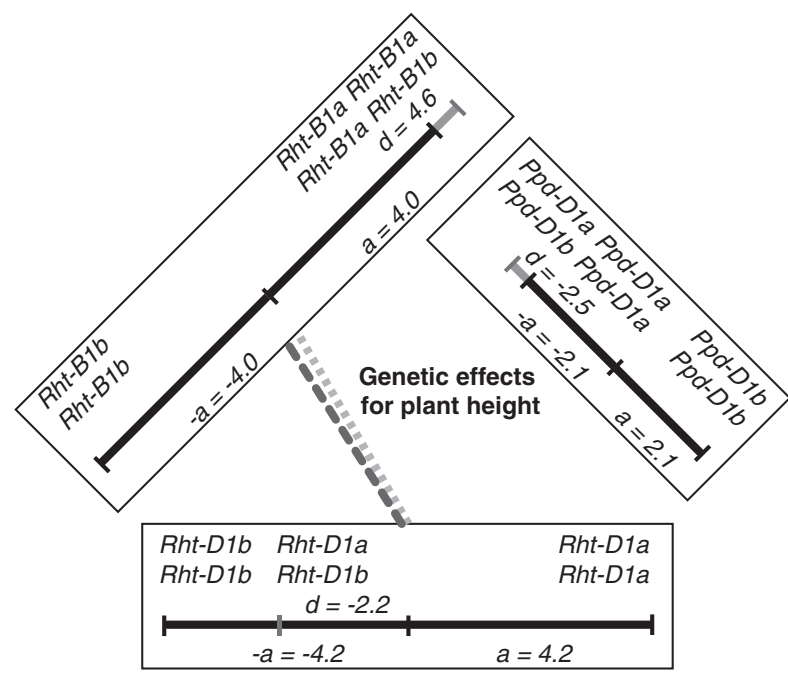

Epistatic interactions involving additiv (a) effects " $\ldots$ Epistatic interactions involving additiv (a) and dominance (d) effects

Figure 3 Genetic effects for plant height for $P p d-D 1$, Rht-B1 and Rht-D1 genotypes as determined in a population of 135 parent wheat lines and their 1604 single-cross hybrids. 

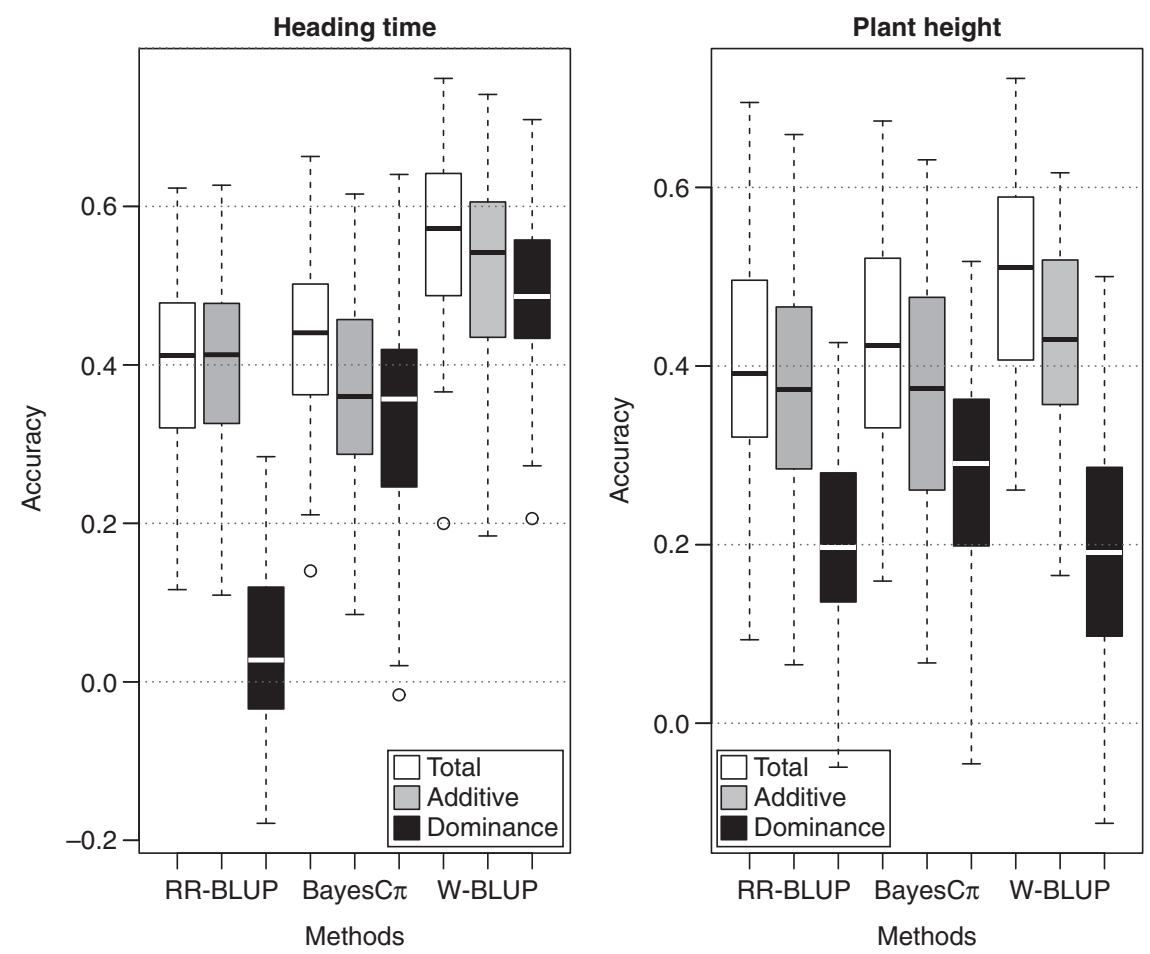

Figure 4 Box-and-whisker plots of accuracy to predict heading time and plant height for the three genomic selection methods RR-BLUP, BayesC $\pi$ and W-BLUP based on 1280 SNPs and trait-specific functional markers.

Table 3 Average prediction accuracies of MAS and three different approaches of genomic selection for simulated data sets

\begin{tabular}{|c|c|c|c|c|c|c|}
\hline \multirow[t]{2}{*}{ Method } & \multicolumn{3}{|c|}{$L E-Q T L$} & \multicolumn{3}{|c|}{$L D-Q T L$} \\
\hline & Total & Additive & Dominance & Total & Additive & Dominance \\
\hline $\mathrm{MAS}^{\mathrm{a}}$ & 0.55 & 0.46 & 0.31 & 0.66 & 0.61 & 0.35 \\
\hline RR-BLUPb & 0.46 & 0.39 & 0.34 & 0.75 & 0.67 & 0.46 \\
\hline BayesC $\pi^{\mathrm{b}}$ & 0.58 & 0.47 & 0.36 & 0.76 & 0.66 & 0.43 \\
\hline W-BLUPb & 0.74 & 0.63 & 0.45 & 0.77 & 0.69 & 0.43 \\
\hline
\end{tabular}

Abbreviations: LD, linkage disequilibrium; LE, linkage equilibrium; MAS, marker-assisted selection; QTL, quantitative trait locus; RR-BLUP, ridge regression best linear unbiased prediction; W-BLUP weighted best linear unbiased prediction.

Based on representative single-nucleotide polymorphisms (SNPs) from our study, we assumed a scenario $L E-Q T L$, in which a selected marker was in no LD with all other markers involved, and a scenario $L D-Q T L$, in which a selected marker was in strong LD with at least 10 further markers.

aMAS prediction accuracy according to cross-validation.

bGenomic selection accuracies according to cross-validation for RR-BLUP, BayesC $\pi$ and W-BLUP.

parental inbred lines besides the hybrids to estimate the marker effects. Obviously, this contributed to the precision to estimate marker effects.

The genotypic variation of heading time and plant height was further predicted based on 1280 SNP markers in combination with functional markers by three different genomic selection approaches, including the well-established RR-BLUP and BayesC $\pi$ methods as well as the newly developed W-BLUP. Cross-validation studies of predicted values revealed that for most scenarios accuracies based on additive and dominance effects were higher than that based on additive or dominance effects alone for both traits (Figure 4). An interesting exception is made by the very low accuracies based on dominance effects observed for heading time and RR-BLUP that are in sharp contrast to the results from BayesC $\pi$ and W-BLUP. The differences in accuracies can be explained by the large contribution to the phenotypic variation of the dominance effect of $P p d-D 1$ that can only be properly handled by low shrinkage parameters for this marker. In the following, we will present only the prediction accuracies for combined additive and dominance effects.

Accuracies of prediction from marker-assisted selection as well as accuracies of prediction from all three genomic selection approaches differed substantially between the two traits of interest (Table 2). For heading time, prediction accuracy decreased from 0.54 with marker-assisted selection to 0.40 with RR-BLUP ( $-25 \%)$, and 0.44 with BayesC $\pi(-17 \%)$, and only improved to 0.58 with W-BLUP $(+7 \%)$. In contrast, for plant height, the low accuracy of 0.38 seen for marker-assisted selection gradually improved over 0.39 with RR-BLUP $(+4 \%)$ and 0.42 with BayesC $\pi(+10 \%)$ to 0.50 with W-BLUP $(+24 \%)$. However, even with W-BLUP, accuracy of prediction stayed lower for plant height compared with heading time.

\section{Comparison of marker-assisted and genomic selection based on} simulations

Although $P p d-D 1$ controlling heading date was found in LD $\left(r^{2}=0.15\right)$ with only one SNP marker, Rht-B1, Rht-D1 and Ppd-D1 relevant for plant height were found in total to be in $\mathrm{LD}$ with $16 \mathrm{SNP}$ markers. In order to test this as an explanation for the different predictive powers of genomic selection based on combined genomewide and functional marker information for different traits, we performed a simulation study contrasting a scenario in which functional SNPs of interest were in linkage equilibrium with the panel of markers (LE-QTL) with one in which functional SNPs were in $\mathrm{LD}$ with several markers $(L D-Q T L)$. This simulation scenario revealed that the pattern of LD between functional SNPs with a substantial contribution to the genotypic variance is crucial for the 
accuracy of genomic selection approaches (Table 3). Looking at prediction accuracies for combined additive and dominance effects, we observed for the $L E$-QTL scenario a decrease in prediction accuracy from 0.55 for marker-assisted selection to 0.46 for RR-BLUP $(-16 \%)$, but an increased accuracy with 0.58 for BayesC $\pi(+5 \%)$ and 0.78 for W-BLUP $(+42 \%)$. Using the preknowledge on important functional markers in the W-BLUP approach led to considerably improved prediction accuracy as compared with marker-assisted selection in this scenario. For the $L D-Q T L$ scenario, we also observed, although less pronounced, an increase in prediction accuracy when shifting from marker-assisted selection with 0.66 to genomic selection with close to 0.76 for all three approaches $(+15 \%)$ irrespective of the method applied.

\section{DISCUSSION}

Development of hybrid breeding opens a new route to increase yield potential in wheat, but it is also connected with new challenges, the appropriate balancing of heading time and plant height being two of them (Longin et al., 2012). Heading time and plant height are traits important for performance of winter wheat and thus have received substantial attention in line breeding. Key gene loci controlling heading time $(P p d-D 1)$ and plant height (Rht-B1, Rht-D1 and PpdD1) are well known, and favorable alleles have been pivotal in shaping current elite wheat (Worland et al., 1998; Peng et al., 1999; Beales et al., 2007). However, more QTLs with small to medium effects are to be expected to fine-tune these traits in both inbred lines and hybrids. Thus, we obtained heading time and plant height phenotypic data in field trials at four locations and generated genotyping data using a 9k wheat SNP array and functional marker tests for $P p d-D 1$, $R h t-B 1$ and $R h t-D 1$ for a population of 135 wheat inbred lines and 1604 hybrids derived from them.

Unexpectedly, marker-assisted selection as well as genomic selection approaches RR-BLUP and BayesC $\pi$ showed quite different accuracies of prediction for the two traits of interest in cross-validation tests. For heading time, prediction by marker-assisted selection based on $P p d-D 1$ alone was already highly accurate for inbred lines, hybrids and the combination of both. In contrast, for plant height, prediction by marker-assisted selection based on Rht-B1, Rht-D1 and Ppd-D1 was accurate only for inbred lines alone, but not for hybrids alone or the combination of inbred lines and hybrids. Cross-validation fully confirmed the high accuracy of prediction by marker-assisted selection for heading time, but indicated that the already lower accuracy of prediction for plant height was slightly overestimated. Genomic selection also showed different accuracies of prediction for the two traits in cross-validation. In comparison with marker-assisted selection, accuracy of prediction of heading time dwindled in genomic selection with RR-BLUP and was still low with BayesC $\pi$ for combined inbred lines and hybrids, whereas for plant height, it improved with RR-BLUP and even further with BayesC $\pi$. Consequently, the advantage of marker-assisted versus genomic selection strongly depends on the trait of interest and the genetic architecture underlying it.

One further explanation for the differences in prediction accuracies of genomic selection for the two traits might be differences in the LD structure. Whereas $P p d-D 1$ controlling heading date is in LD with only one SNP marker, Rht-B1, Rht-D1 and $P p d-D 1$ relevant for plant height are all together in LD with a total of 16 SNP markers. Such an influence of LD was confirmed by our simulation studies. In a setup assuming a functional marker without SNP markers in LD, accuracies of prediction of marker-assisted selection, RR-BLUP and BayesC $\pi$ showed a drop with RR-BLUP similar to that seen for heading time, whereas in a setup assuming a functional marker in LD with several
SNP markers, increasing accuracies similar to that seen for plant height were found.

Yet another reason for the differences in the accuracies of prediction between the two traits might reside in hybrid-related effects. For both heading time and plant height, additive as well as dominance effects contributed to the accuracies of genomic selection, but to different extents. Additive effects were found most important for heading time, in particular in context with RR-BLUP, whereas for plant height, additive and dominance effects contributed in a more balanced way. This is consistent with the very low midparent heterosis for heading time and the contrasting substantial midparent heterosis for plant height observed in our study.

With regard to the control of heading time and plant height, partial dominance of the $P p d-D 1$ allele promoting early heading $(P p d-D 1 a)$ and of the Rht-D1 allele promoting short plant stature (Rht-D1b) reported by Worland et al. (1998) and Beales et al. (2007) could be confirmed. In contrast, the apparent overdominance of alleles $P p d-D 1 a$ and Rht-B1a (the latter one even in the direction of tall plant stature) in context with plant height found in our study is inconsistent with these publications. However, there are previous reports of similar overdominance (Allan et al., 1968) and generally increased plant height (Allan et al., 1968; Fick and Qualset, 1973) in wheat $F_{1}$ hybrids. Furthermore, because of the need to use late-heading tall inbred lines as male and early-heading shorter ones as female parents in wheat hybrid production, $P p d-D 1, R h t-B 1$ and $R h t-D 1$ alleles promoting early heading $(P p d-D 1 a)$ and short stature ( $P p d-D 1 a$, $R h t-B 1 b$ and $R h t-D 1 b)$ were distributed nonrandomly among the parental lines, and thus also among the hybrids.

The nonsatisfactory performance of RR-BLUP and also BayesC $\pi$ in the prediction of heading time and plant height is in part because of inappropriate weighting of functional marker versus SNP marker contributions. To resolve this limitation, we developed W-BLUP that balances the contributions from random and functional markers by including additional effect of the latter ones. Accordingly, W-BLUP performed better than both RR-BLUP and BayesC $\pi$ in prediction based on experimental as well as simulated data. Thus, W-BLUP provides a new robust tool to bridge the gap between marker-assisted and genomic selection.

Heading time and plant height also differed in their levels of heterosis, with heading time showing almost no response to hybridization, whereas plant height was increased by 4 to $15 \%$. The difference in the response of the two traits to hybridization and the substantial heterosis in plant height toward tall plant stature had already shown up in the initial analysis of the data set (Longin et al., 2013). As a short, sturdy plant stature is favorable in wheat cultivation (Borlaug, 1983), a major challenge for future wheat hybrid breeding will be to counterbalance the heterosis in plant height and to mobilize the surplus in biomass into grain yield. In previous conventional wheat line breeding, plant height has been traded for yield gain by using dwarfing alleles $R h t-B 1 b$ or $R h t-D 1 b$, usually either one or the other, but rarely both in combination, as homozygosity for both alleles leads to very small plants with diminished yield potential.

As the degree of heterosis in plant height seemed to be influenced by the $R h t-B 1$ and $R h t-D 1$ alleles present, with the already tall Rht-B1a; Rht-D1a homozygous setup allowing less heterosis than the shorter Rht-B1b;Rht-D1a setup, plant height heterosis in hybrids might actually be able to mend the drawbacks of even a Rht-B1b; $R h t-D 1 b$ double-homozygous setup. A respective tendency toward increased plant height has been described for wheat hybrids obtained from crosses of semi-dwarf (likely Rht-B1b;Rht-D1b) lines previously (Allan et al., 1968). However, the single Rht-B1b; Rht-D1b double- 
homozygous inbred line included as a female parent in our study had the lowest grain yield of all inbred lines, ranking 135th among 135 lines, and it would be hard to produce Rht-B1b;Rht-D1b doublehomozygous wheat hybrids on a large scale in practical terms, as the male parents need to be tall to be efficient as pollen donors. A practicable solution might be the generation of hybrids homozygous for $R h t-B 1 b$ and heterozygous for $R h t-D 1 b$ by crossing an $R h t-B 1 b ; R h t-D 1 a$ father and an Rht-B1b;Rht-D1b mother. In the one case available to us, the success of this approach was limited. Although the semi-dwarf Rht-B1b;Rht-D1b homozygous inbred line used as female parent had the lowest grain yield of all 135 lines in the study and the tall Rht-B1b; Rht-D1a homozygous inbred line used as male parent ranked 11th of all 135 lines with regard to grain yield, the resulting hybrid homozygous for Rht-B1b and heterozygous for $R h t-D 1 b$ had a plant height within the appropriate range and ranked 1478th of 1604 hybrids with regard to grain yield, indicating a grain yield midparent heterosis of $12.3 \%$ (data not shown). Thus, although this one observation seems rather promising, the balancing of plant height heterosis in hybrid wheat is still providing a substantial challenge.

\section{DATA ARCHIVING}

Original data are included as Supplementary Table S1 in form of a Microsoft Excel file and are available from the Dryad Digital Repository: doi: 10.5061/dryad.461nc.

\section{CONFLICT OF INTEREST}

The authors declare no conflict of interest.

\section{ACKNOWLEDGEMENTS}

We thank Andreas Börner for critical comments on the manuscript. This research was conducted within the HYWHEAT project funded by BMBF (Grant ID: FKZ0315945D).

Akhunov E, Nicolet C, Dvorak J (2009). Single nucleotide polymorphism genotyping in polyploid wheat with the Illumina GoldenGate assay. Theor Appl Genet 119: 507-517.

Allan RE, Vogel OA, Peterson CJ (1968). Inheritance and differentiation of semidwarf culm length of wheat. Crop Sci 8: 701-704.

Beales J, Turner A, Griffiths S, Snape JW, Laurie DA (2007). A pseudo-response regulator is misexpressed in the photoperiod insensitive Ppd-Dla mutant of wheat (Triticum aestivum L.). Theor App/ Genet 115: 721-733.

Bernardo R (2001). What if we knew all the genes for a quantitative trait in hybrid crops? Crop Sci 41: 1-4

Bernardo R (2008). Molecular markers and selection for complex traits in plants: learning from the last 20 years. Crop Sci 48: 1649-1664.

Borlaug NE (1983). Contributions of conventional plant breeding to food production. Science 219: 689-693.

Butler D, Cullis BR, Gilmour AR, Gogel BJ (2009). ASREML-R, Reference Manual, Version 3. Queensland Department of Primary Industries and Fisheries: Brisbane.

Cavanagh CR, Chao S, Wang S, Huang BE, Stephen S, Kiani S et al. (2013). Genome-wide comparative diversity uncovers multiple targets of selection for improvement in hexaploid wheat landraces and cultivars. Proc Natl Acad Sci USA 110: 8057-8062.

Clark SA, Hickey JM, van der Werf JHJ (2011). Different models of genetic variation and their effect on genomic evaluation. Genet Sel Evol 43: 18.

Crossa J, de los Campos G, Perez P, Gianola D, Burgueno J, Araus JL et al. (2010). Prediction of genetic values of quantitative traits in plant breeding using pedigree and molecular markers. Genetics 186: 713-U406.

Dekkers J, Garrick D, Fernando R (2009). Use of high-density SNP genotyping for genetic improvement of livestock. A Short Course Organized by the Animal Breeding \& Genetics Department of Animal Science. Iowa State University: Ames, http://www.ans.iastate. edu/stud/courses/short/2009/

Distelfeld A, Li C, Dubcovsky J (2009). Regulation of flowering in temperate cereals. Curr Opin Plant Biol 12: 178-184.
Ellis H, Spielmeyer W, Gale R, Rebetzke J, Richards A (2002). 'Perfect' markers for the Rht-B1b and Rht-D1b dwarfing genes in wheat. Theor App/ Genet 105: 1038-1042.

Falconer DS, Mackay TF (1996). Introduction to Quantitative Genetics, 4th edn Longman: Harlow.

Fick GN, Qualset CO (1973). Genes for dwarfness in wheat, Triticum aestivum L. Genetics 75: 531-539.

Goddard ME, Hayes BJ (2009). Mapping genes for complex traits in domestic animals and their use in breeding programmes. Nat Rev Genet 10: 381-391.

Guo ZA, Song YX, Zhou RH, Ren ZL, Jia JZ (2010). Discovery, evaluation and distribution of haplotypes of the wheat Ppd-D1 gene. New Phytol 185: 841-851.

Habier D, Fernando RL, Kizilkaya K, Garrick DJ (2011). Extension of the bayesian alphabet for genomic selection. BMC Bioinformatics 12: 186

Hallauer AR, Miranda JB (1988). Quantitative Genetics in Maize Breeding, 2nd edn. lowa State University Press: Ames.

Heffner EL, Sorrells ME, Jannink JL (2009). Genomic selection for crop improvement. Crop Sci 49: 1-12.

Henderson C (1984). Application of Linear Models in Animal Breeding. University of Guelph: Ontario.

Heslot N, Yang HP, Sorrells ME, Jannink JL (2012). Genomic selection in plant breeding: a comparison of models. Crop Sci 52: 146-160.

Le Couviour F, Faure S, Poupard B, Flodrops Y, Dubreuil P, Praud S (2011). Analysis of genetic structure in a panel of elite wheat varieties and relevance for association mapping. Theor Appl Genet 123: 715-727.

Longin CF, Gowda M, Mühleisen J, Ebmeyer E, Kazman E, Schachschneider R et al. (2013). Hybrid wheat: quantitative genetic parameters and consequences for the design of breeding programs. Theor App/ Genet 126: 2791-2801.

Longin CF, Muhleisen J, Maurer HP, Zhang H, Gowda M, Reif JC (2012). Hybrid breeding in autogamous cereals. Theor Appl Genet 125: 1087-1096.

Massman JM, Jung HJG, Bernardo R (2013). Genomewide selection versus markerassisted recurrent selection to improve grain yield and stover-quality traits for cellulosic ethanol in maize. Crop Sci 53: 58-66.

Meuwissen TH, Hayes BJ, Goddard ME (2001). Prediction of total genetic value using genome-wide dense marker maps. Genetics 157: 1819-1829.

Miedaner T, Hubner M, Korzun V, Schmiedchen B, Bauer E, Haseneyer G et al. (2012). Genetic architecture of complex agronomic traits examined in two testcross populations of rye (Secale cereale L.). BMC Genomics 13: 706.

Möhring J, Piepho HP (2009). Comparison of weighting methods in two-stage analysis of plant breeding trials. Crop Sci 49: 1977-1988.

Peng JR, Richards DE, Hartley NM, Murphy GP, Devos KM, Flintham JE et al. (1999). 'Green revolution' genes encode mutant gibberellin response modulators. Nature 400 : 256-261.

Perez P, de los Campos G, Crossa J, Gianola D (2010). Genomic-enabled prediction based on molecular markers and pedigree using the Bayesian linear regression package in $\mathrm{R}$. Plant Genome 3: 106-116.

R Core Team (2012). R: A Language and Environment for Statistical Computing. R Foundation for Statistical Computing: Vienna, http://www.R-project.org/

Riedelsheimer C, Endelman JB, Stange M, Sorrells ME, Jannink JL, Melchinger AE (2013). Genomic predictability of interconnected biparental maize populations. Genetics 194: 493-503.

Seki M, Chono M, Matsunaka H, Fujita M, Oda S, Kubo K et al. (2011). Distribution of photoperiod-insensitive alleles $P p d-B 1 a$ and $P p d-D 1 a$ and their effect on heading time in Japanese wheat cultivars. Breed Sci 61: 405-412.

Stram DO, Lee JW (1994). Variance-components testing in the longitudinal mixed effects model. Biometrics 50: 1171-1177

Turner A, Beales J, Faure S, Dunford RP, Laurie DA (2005). The pseudo-response regulator $\mathrm{Ppd}-\mathrm{H} 1$ provides adaptation to photoperiod in barley. Science 310: 1031-1034.

Venables WN, Ripley BD (2002). Modern Applied Statistics with S, 4th edn Springer: New York.

Whittaker JC, Thompson R, Denham MC (2000). Marker-assisted selection using ridge regression. Genet Res 75: 249-252.

Wilhelm EP, Boulton MI, AI-Kaff N, Balfourier F, Bordes J, Greenland AJ et al. (2013a). Rht-1 and Ppd-D1 associations with height, GA sensitivity, and days to heading in a worldwide bread wheat collection. Theor App/ Genet 126: 2233-2243.

Wilhelm EP, Mackay IJ, Saville RJ, Korolev AV, Balfourier F, Greenland AJ et al. (2013b). Haplotype dictionary for the Rht-1 loci in wheat. Theor App/ Genet 126: 1733-1747.

Williams E, Piepho HP, Whitaker D (2011). Augmented p-rep designs. Biometrical J 53: 19-27.

Worland AJ, Börner A, Korzun V, Li WM, Petrovic S, Sayers EJ (1998). The influence of photoperiod genes on the adaptability of European winter wheats (Reprinted from Wheat: Prospects for global improvement, 1998). Euphytica 100: 385-394.

Würschum T, Langer SM, Longin CF, Korzun V, Akhunov E, Ebmeyer E et al. (2013). Population structure, genetic diversity and linkage disequilibrium in elite winter wheat assessed with SNP and SSR markers. Theor App/ Genet 126: 1477-1486.

Zhao YS, Gowda M, Würschum T, Longin CF, Korzun V, Kollers S et al. (2013b). Genetic architecture of frost tolerance in wheat. $J$ Exp Bot 64: 4453-4460.

Zhao YS, Zeng J, Fernando R, Reif JC (2013a). Genomic prediction of hybrid wheat performance. Crop Sci 53: 802-810.

Supplementary Information accompanies this paper on Heredity website (http://www.nature.com/hdy) 